\title{
Adalimumab induced chronic interstitial nephritis: A controlled blinded trial in mice
}

Background: Adalimumab is a known drug modifying agent for rheumatoid arthritis (RA) and juvenile idiopathic arthritis (JIA) treatment. However little is known about the risk of nephrotoxicity. To determine whether the inhibition of tumor necrosis factor (TNF) binding to cell-surface TNF receptor sites by adalimumab result in acute nephritis in $B A L B / c$ mice.

Method: Twenty Balb/c mice were studied. Ten mice received subcutaneous (SC) injection of adalimumab $(0.0175 \mathrm{mg}$ per $25 \mathrm{~kg}$ body weight per $0.03 \mathrm{ml}$ normal saline) biweekly $(0,2,4,6$ and 8 weeks). For the control group, 10 mice received a SC injection of normal saline (NS) $(0.03 \mathrm{ml})$. At the 10 th week post injection, the mice were sacrificed, and histopathological studies were conducted.

Results: Of the adalimumab-treated group, 4/10 mice died. Kidney histology for the adalimumab-treated group showed that $6 / 6$ displayed histopathological changes manifested as patchy chronic interstitial inflammation with focal tubular epithelial cells vaculation. In contrast, only minimal inflammation was observed in 4/10 mice in the control group $(p=0.01)$.

Conclusion: Adalimumab may play a role in inducing chronic interstitial nephritis.

Keywords: adalimumab • interstitial nephritis $\bullet$ mice $\bullet$ histology

\section{Introduction}

Tumor necrosis factor-alpha (TNF- $\alpha$ ) is a major pro inflammatory cytokine in many chronic inflammatory diseases, including rheumatoid arthritis (RA), juvenile idiopathic arthritis (JIA), ankylosing spondylitis, psoriatic arthritis and Crohn's disease [1-3]. Anti TNF- $\alpha$ agents used in the management of RA and other rheumatologic diseases include etanercept, a soluble TNF- $\alpha$ receptor; infliximab, a chimeric monoclonal IgG I anti TNF- $\alpha$ antibody; and adalimumab, a fully humanized monoclonal antibody [3]. These agents are generally well tolerated, the most common side effects being headache, injection site reaction, upper respiratory tract infection and diarrhea [4]. Severe side effects including serious infection, neoplasia, heart failure, central nervous system involvement and hematologic disorders are rare $[4,5]$.

More than 1500 cases of autoimmune disease induced by biologics have been reported, including a wide variety of both systemic (lupus, vasculitis, sarcoidosis, antiphospholipid syndrome and inflammatory myopathies) and organ specific (interstitial lung disease, uveitis, optic neuritis, peripheral neuropathies, multiple sclerosis, psoriasis, inflammatory bowel disease and autoimmune hepatitis) autoimmune processes. These processes are overwhelmingly associated with anti-TNF agents [6-13].

Drug Renal toxicities are rare secondary to anti TNF agents and may present as glomerulonephritis and interstitial nephritis [14].

Acute interstitial nephritis (AIN) is a common cause of acute kidney injury. Many etiologies of AIN have been recognized including allergic/ drug-induced, infectious, 4 autoimmune/ systemic and idiopathic forms of disease. The most common etiology of AIN is drug induced disease, which is thought to underlie $60-70 \%$ of cases [15-17].

The development of anti TNF-induced renal disease remain a matter of concern. The incidence of such advance effects is unknown. The mechanism of anti TNF alpha induced renal disease and autoimmunity remain unknown [14].
Sultan M. Al Mogairen*

Department of Medicine, Faculty of Medicine, King Saud University, Saud Arabia

*Author for correspondence:

almogairin@gmail.com 
To the best of our knowledge there is no controlled animal histopathological study demonstrating the effect of adalimumab on the kidney.

The aim of current study to demonstrate whether the subcutaneous adalimumab will result in acute or chronic renal injury in BALB/c mice.

\section{Methods}

Twenty BALB/c male mice (average weight of 25 g) were purchased from the Faculty of Pharmacy at King Saud University, (Kingdom of Saudi Arabia).

Mice were kept in polycarbonate metrolon plastic cages covered with a stainless-steel cover in the animal house at the College of Medicine at King Saud University, Riyadh, Saudi Arabia. The mice were exposed to $12 \mathrm{~h}$ of darkness and $12 \mathrm{~h}$ of light daily, and were kept under observation for 3 weeks. No evidence of sickness was observed. All mice were 12-14 weeks old at the start of the experiment.

Twenty mice were divided into two groups that each consisted of ten mice. One group received SC injections of Adalimumab $(0.0175 \mathrm{mg} / 0.03$ $\mathrm{ml}$ NS) biweekly at $0,2,4,6$ and 8 weeks. The second group received a SC injection of normal saline $(0.03 \mathrm{ml})$ weekly. We used weekly NS injection for this common control group because this study is considered as a part of multi-arm animal trials.

Since the appropriate dosage for SC injections of adalimumab has not been reported in animals, we extrapolated this based upon recommended doses used in human studies $(0.8 \mathrm{mg} / \mathrm{kg})$ for the treatment of severe chronic plaque psoriasis in children and adolescents, but we used less than the recommended dosage $(0.7 \mathrm{mg} / \mathrm{kg})$ to improve the safety of the drug [1].

During the 10th week, mice were sacrificed by applying pressure to the neck and dislocating the cervical spine. Subsequently kidney histopathological studies were performed for each mouse in both groups. The kidney tissues were bisected and fixed in $10 \%$ buffered formalin for $24 \mathrm{~h}$. The tissues were then processed using hematoxylin and eosin (H/E) stain. The slides were examined using a light microscope. Evaluation of liver tissue slides were performed blindly by a histopathologist.

\section{Statistical analysis}

Statistical differences between the Adalimumab- treated and saline-treated groups were calculated using Fisher's exact test. A p-value $<0.05$ was considered significant.

\section{Results}

Of the Adalimumab-treated mice, 4/10 died during the last 3 weeks of experiment. There was no clinical evidence of sickness prior to death. No deaths were reported in the control group.

Histopathological results are shown for the normal saline control group Table 1 and the adalimumab-treated group Table 2. Kidney changes were negative if all the histological features were normal Figure $1 \mathrm{~A}$ and $1 \mathrm{~B}$. Otherwise they remained positive. Positive kidney tissues were observed in 6/6 mice treated with SC injections of abatacept group Table 2 and Figure 2A-C. In contrast, positive mild kidney changes were observed in $4 / 10$ mice in the control group $(\mathrm{p}=0.0 .1)$.

\section{Discussion}

TNF- $\alpha$ antagonists are effective in the treatment of chronic inflammatory joint disease. Despite a good overall safety profile, they can induce a number of adverse effects, including autoimmunity and infections [6-17].

A nationwide survey was conducted between December 2004 and January 2005, a nationwide survey was conducted among 1200 hospitalbased rheumatologists and internists in France, who were asked to report cases of vasculitis in patients taking TNF- $\alpha$ antagonists. The survey identified 39 cases (32 women) of vasculitis during TNF- $\alpha$ antagonists therapy. The joint disease was rheumatoid arthritis (RA) in 34 patients, juvenile idiopathic arthritis in two patients, ankylosing spondylitis in two patients, and psoriatic arthritis in one patient. The TNF- $\alpha$ antagonists was etanercept in 21 patients, infliximab in 15 , adalimumab in 2 , and another drug in 1 ; mean treatment duration was 9.6 months. The manifestations of vasculitis involved the skin $(n=32)$; peripheral nervous system ( $n=9)$; kidney $(n=7)$; central nervous system $(n=3)$; pleura $(n=2)$, pericardium $(n=2)$; and the lung, gallbladder, and heart $(n=1$ each). Histology (30 biopsies from 27 patients) showed no necrotizing vasculitis in 12 patients, necrotizing vasculitis in 7 , an inflammatory dermal infiltrate without vasculitis in 3, extravascular necrotic granulomas in 2, chilblain lupus in 1, and cicatricial, fibro-inflammatory changes in 1. Renal biopsy in three patients showed extra capillary glomerulonephritis with 


\begin{tabular}{|c|c|c|c|c|c|c|c|}
\hline Mouse No. & Glomeruli & $\begin{array}{c}\text { Acute tubular injury } \\
\text { reactive atypia }\end{array}$ & TV & Acute TII & Chronic TII & IF / TA & BV \\
\hline S-1 & 0 & 0 & 0 & 0 & 0 & 0 & 0 \\
\hline S-2 & 0 & 0 & 0 & 0 & 0 & 0 & 0 \\
\hline S-3 & 0 & 0 & 0 & 0 & 0 & 0 & 0 \\
\hline S-4 & 0 & 0 & 0 & 0 & 0 & 0 & 0 \\
\hline S-5 & 0 & 0 & 0 & 0 & 0 & 0 & 0 \\
\hline S-6 & 0 & 0 & 1 & 0 & 0 & 0 & 0 \\
\hline S-7 & 0 & 0 & 0 & 0 & 0 & 0 & 0 \\
\hline S-8 & 0 & 0 & 0 & 0 & 1 & 0 & 0 \\
\hline S-9 & 0 & 0 & 1 & 0 & 0 & 0 & 0 \\
\hline S-10 & 0 & 0 & 1 & 0 & 1 & 0 & 0 \\
\hline \multicolumn{8}{|c|}{$\begin{array}{l}\text { ABBREVIATIONS } \\
\text { TV=tubular vacuolization } \\
\text { TA=tubular atrophy } \\
\text { TIl=tubulointerstitial inflammation } \\
\text { TECs=tubular epithelial cells } \\
\text { IF/TA=interstitial fibrosis / tubular atrophy } \\
\text { BV=blood vessels }\end{array}$} \\
\hline \multicolumn{8}{|c|}{$\begin{array}{l}\text { KEY } \\
0=\text { normal : } 0 \\
1=\text { Mild }: 1 \text { to } 25 \% \\
2=\text { Moderate }: 26 \text { to } 50 \% \\
3=\text { Severe }:>50 \%\end{array}$} \\
\hline
\end{tabular}

\begin{tabular}{|c|c|c|c|c|c|c|c|}
\hline Mouse No. & Glomeruli & $\frac{\text { Acute tubular injury }}{\text { reactive atypia }}$ & TV & Acute TII & Chronic TII & IF / TA & BV \\
\hline SP 15-D1 & 0 & 0 & 1 & 0 & 0 & 0 & 0 \\
\hline SP 15-D2 & 0 & 1 & 1 & 0 & 1 & 0 & 0 \\
\hline SP 15-D3 & 0 & 0 & 1 & 0 & 1 & 0 & 0 \\
\hline SP 15-D4 & 0 & 0 & 1 & 0 & 1 & 0 & 0 \\
\hline SP 15-D5 & 0 & 0 & 1 & 0 & 0 & 0 & 0 \\
\hline SP 15-D6 & 0 & 1 & 1 & 0 & 1 & 0 & 0 \\
\hline \multicolumn{8}{|c|}{$\begin{array}{l}\text { ABBREVIATIONS } \\
\text { TV=tubular vacuolization } \\
\text { TA=tubular atrophy } \\
\text { TII=tubulointerstitial inflammation } \\
\text { TECs=tubular epithelial cells } \\
\text { IF/TA=interstitial fibrosis / tubular atrophy }\end{array}$} \\
\hline \multicolumn{8}{|c|}{$\begin{array}{l}\text { KEY } \\
0=\text { normal : } 0 \\
1=\text { Mild : } 1 \text { to } 25 \% \\
2=\text { Moderate }: 26 \text { to } 50 \% \\
3=\text { Severe }:>50 \%\end{array}$} \\
\hline
\end{tabular}

$\operatorname{IgA}$ deposits $(\mathrm{n}=2)$ or active floccular necrosis against a background of glomerular sclerosis $(\mathrm{n}=1)[18]$.

Nearly 200 cases of systemic autoimmune diseases triggered by biological agents. Over whelming anti-tumor necrosis factor (TNF) more than 140 cases of vasculitis induced by biological agents have been reported. Despite more than 140 cases of lupus induced by biological agents have been reported, less than $40 \%$ of them fulfilled the classification criteria for SLE [7,19].

More cases of anti-tumor factor-alpha induced lupus are caused by infliximab, followed by etanercept and adalimumab [20,21].

Several factors support an etiologic role for anti-TNF agents in the development of vasculitis. Firstly, the occurrence of vasculitis was temporally associated with the initiation of therapy. Secondly, in some patients treated with etanercept, the skin lesions started at the injection site and spread to involve other areas of the body, supporting a role for a direct antigen-mediated hypersensitivity vasculitis. Thirdly, even though patients were reported to 

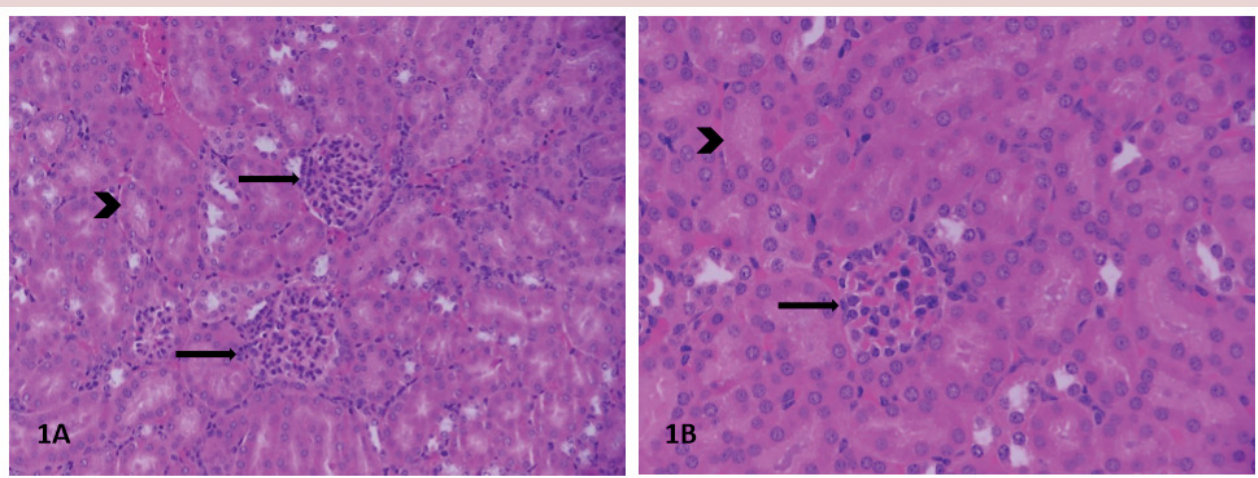

Figure 1A-B. Photomicrograph of control mouse kidney showing a normal glomerulus (arrow) surrounded by normal tubules (arrow head) (hematoxylin and eosin stain, original magnification x200 and x400 respectively)

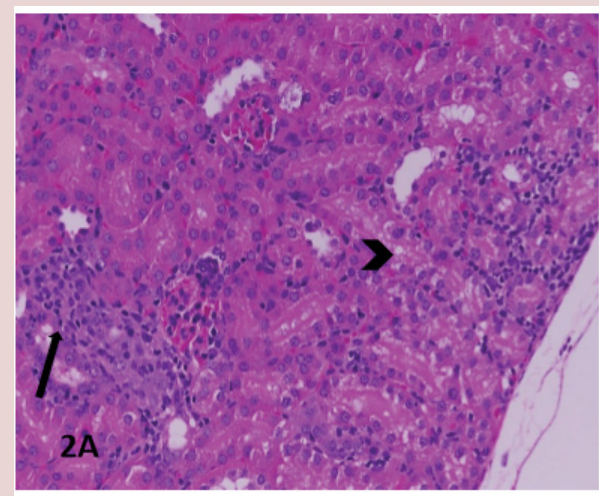

Figure 2A. Photomicrograph of mouse kidney treated with adalimumab shows patchy mild chronic interstitial inflammation in the interstitium (arrows) with associated focal mild tubular vacuolization of the tubular cell cytoplasm (arrow head) (hematoxylin and eosin stain, original magnification $\times 200$ )

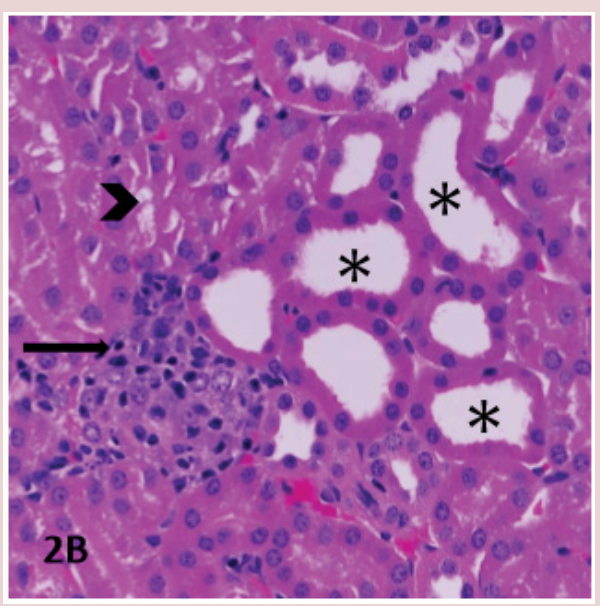

Figure 2B. Photomicrograph of mouse kidney treated with adalimumab shows focal interstitial chronic inflammation (arrow), focal mild acute tubular injury in the adjacent tubules (asterisks) and vacuolization of some of the tubular epithelial cells (arrow head) (hematoxylin and eosin stain, original magnification $\times 400$ )

be on concomitant, medications that have been associated with vasculitis, the cutaneous lesions resolved in $90 \%$ of patients after discontinuation of anti-TNF agents. Lastly, a positive rechallenge phenomenon was seen in two thirds of reported cases $[22,23]$. 


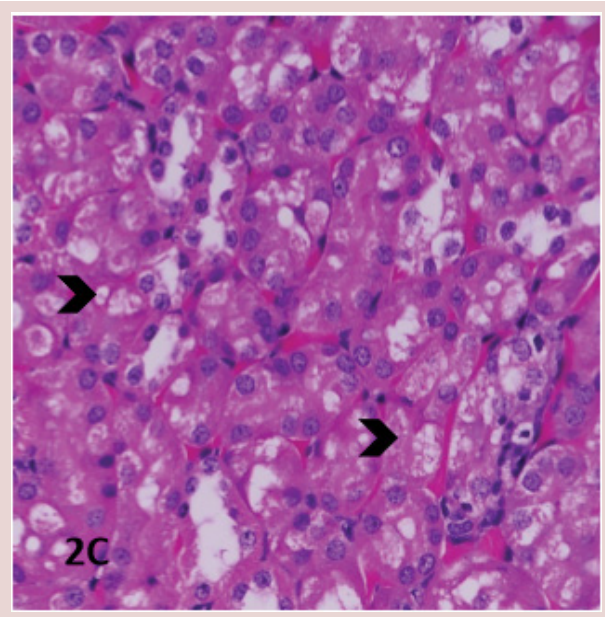

Figure 2C. Photomicrograph of mouse kidney treated with adalimumab shows vacuolization in the cytoplasm of the tubular epithelial cells (arrow heads) (hematoxylin and eosin stain, original magnification $\mathrm{x400)}$

Mortality rates between three TNF inhibitors (infliximab, etanercept and adalimumab) regardless of adjustment and modeling approach for infliximab versus entanercept, HR 1.3 (95\% confidence interval $0.7-1.7)$ and for adalimumab versus entanercept, HR $1.3(95 \%$ confidence interval 0.9-2.0). This result revealed no statistically significant difference in mortality rates between the 3 TNF inhibitors [24]. In this study the mortality in the adalimumab group was $40 \%(16.8 \%$ to $68.7 \%)$ on the other hand mortality of control group $0 \%$.

The husbandry was the same for both groups, and there were no clinical signs of sickness prior to death. The deaths were probably the result of cardiovascular events. Renal biopsies were performed in 10 patients with vasculitis induced by tumor necrosis factor targeted therapies and showed glomerulonephritis (GN) that was classified as pauci-immune crescenting necrotizing glomerulonephritis in seven cases, IgA glomerulonephritis in two cases and coexistence of membranous glomerulonephritis and renal vasculitis in the remaining case [22].

Matteo Piga et al. did a systematic review and cohort study of 707 adult patients affected with Rheumatoid arthritis, ankylosing spondylitis and reported 29 cases of biologics induced autoimmune renal disorders. The biologic drug most frequently associated with development of (AIRD) autoimmune renal disorders was etanercept (15 cases, 51.7\%) followed by adalimumab (9 cases, 31.0\%) and infliximab (3 cases, $10.3 \%$ ) while tocilizumab and abatacept were reported in 1 case $(3.4 \%)$ for each. Thirteen out of $29(44.8 \%)$ cases were classified as affected by isolated autoimmune renal disorders (IARD), $12(411.3 \%)$ as Glomerulonephritis associated with systemic vasculitis (GNSV) and 4 (13.4\%) as Glomerulonephritis in lupus-like syndrome (GNLS) [25].

\section{Conclusion}

To our knowledge this the first experimental controlled study demonstrating adalimumab induced interstitial nephritis in BALB/c mice.

In order to identify those rare individuals with higher risk for development of biologics induced (AIRD), a baseline testing for ANA, anti-dsDNA and ANCA should be performed in all patients prior to starting biologics together with a careful assessment of pre-existing causes of nephropathy, which in our review resulted associated to the development of AIRD. Moreover, during treatment with biologics all patients must undergo at least quarterly evaluation of kidney parameters. If impaired renal function or urinary sediment abnormalities are detected, the possible occurrence of AIRD must be investigated through an accurate clinical and laboratory examination [25].

Although it is unclear whether anti TNF- $\alpha$ agents serve as exacerbating factor of an underlying lupus or triggering factor of SLE, withdrawal of the offending drug remains the mainstay therapy [26].

\section{Acknowledgements}

The author is grateful to the histopathologist Dr. S. Husain for the reporting of the histopathological studies and the pharmacists Mr. Wajed Ali Khan, Mr. Mohammed Ali Khan and Mr. Nazeeh Al 
Saeed. The author is also grateful to Dr. Najma Khalil, Mr. Amir Marzouk, Mr. Saleh Abu-AlGhaith, Ms. N. Ocampo and Ms. J. Mendoza for their cooperation, and to the Research Ethics Committee at the College of Medicine for the approval of this study.

\section{Conflict of interest}

The author declares no conflict of interest with any institution or /organization.

\section{Funding}

This work was supported by the Deanship of Scientific Research at King Saud University through research group no RGP-126. The funder did not have any role in the study design, data collection and analysis, decision to publish or the preparation of manuscript.

\section{References}

1. Burness $\mathrm{CB}$, McKeage K. Adalimumab: A Review in chronic plaque psoriasis. Drugs. 75, 2119-30 (2015).

2. Monaco C, Nanchahal J, Taylor P et al. Anti-TNF therapy: past, present and future. Int. Immunol. 27, 55-62 (2015)

3. Silva LC, Ortigosa LC, Benard G. Anti-TNF- $\alpha$ agents in the treatment of immune-mediated inflammatory diseases: mechanisms of action and pitfalls. Immunotherapy. 2, 817-33 (2010).

4. Khanna D, McMahon M, Furst DE. Safety of tumour necrosis factor alpha antagonists. Drug. Saf. 27, 307-24 (2004).

5. Wiens A, Venson R, Correr CJ et al. Meta-analysis of the efficacy and safety of adalimumab, etanercept, and infliximab for the treatment of rheumatoid arthritis. Pharmacotherapy. 30, 339-353 (2010).

6. Perez-Alvarez R, Perez-de-Lis M, Ramos-Casals M et al. Biologics-induced autoimmune diseases. Curr. Opin. Rheumatol. 25, 56-64 (2013).

7. Ramos-Casals M, Alvarez RP, Diaz-Lagares C et al. Autoimmune diseases induced by biological agents: a double-edged sword? Autoimmun. Rev. 9, 188-93 (2010).

8. Beigel F, Schnitzler F, Laubender PR et al. Formation of antinuclear and double-strand DNA antibodies and frequency of lupus-like syndrome in anti-TNF- $\alpha$ antibody-treated patients with inflammatory bowel disease. Inflamm. Bowel. Dis. 17, 91-8 (2011).

9. Seve P, Varron L, Broussolle C et al. Sarcoid-related uveitis occurring during adalimumab therapy. Ocul. Immunol. Inflamm. 20, 59-60 (2012).

10. Perez-Alvarez R, Perez-de-Lis M, Diaz-Lagares C et al. Interstitial lung disease induced or exacerbated by TNFtargeted therapies: analysis of 122 cases. Semin. Arthritis. Rheum. 41, 256-64 (2011).

11. Czaja AJ. Drug-induced autoimmune-like hepatitis. Dig. Dis. Sci. 56, 958-76 (2011).
12. Van Dijken TD, Vastert SJ, Gerloni VM et al. Development of inflammatory bowel disease in patients with juvenile idiopathic arthritis treated with etanercept. J. Rheumatol. 38, 1441-6 (2011).

13. Cullen G, Kroshinsky D, Cheifetz AS et al. Psoriasis associated with anti-tumour necrosis factor therapy in inflammatory bowel disease: a new series and a review of 120 cases from the literature. Aliment. Pharmacol. Ther. 34, 1318-27 (2011).

14. Stokes MB, Foster K, Markowitz GS et al. Development of glomerulonephritis during anti-TNF-alpha therapy for rheumatoid arthritis. Nephrol. Dial. Transplant. 20, 1400-6 (2005).

15. Perazella MA, Markowitz GS. Drug-induced acute interstitial nephritis. Nat. Rev. Nephrol. 6, 461-70 (2010).

16. Clarkson MR, Giblin L, O'Connell FP et al. Acute interstitial nephritis: clinical features and response to corticosteroid therapy. Nephrol. Dial. Transplant. 19, 2778-83 (2004).

17. Baker RJ, Pusey CD. The changing profile of acute tubulointerstitial nephritis. Nephrol. Dial. Transplant. 19, 8-11(2004).

18. Saint Marcoux B, De Bandt M, CRI (Club Rhumatismes et Inflammation). Vasculitides induced by TNF alpha antagonists: a study in 39 patients in France. Joint. Bone. Spine. 73, 710-3 (2006).

19. Ramos-Casals M, Brito-Zeron P, Munoz S et al. A systematic review of the off-label use of biological therapies in systemic autoimmune diseases. Medicine. 87, 345-64 (2008).

20. Subramanian S, Yajnik V, Sands BE et al. Characterization of patients with infliximab-induced lupus erythematosus and outcomes after retreatment with a second anti-TNF agent. Inflamm. Bowel. Dis. 17, 99-104 (2011).

21. Puertas-Abreu E, Polanco ER, Azocar M et al. Onset of lupus like syndrome in patients with spondyloarthritis treated with anti-TNF- $\alpha$. Int. Arch. Med.15, 5-7 (2012).

22. Ramos-Casals M, Brito-Zeron P, Cuadrado MJ et al. Vasculitis induced by tumor necrosis factor-targeted therapies. Curr. Rheumatol. Rep. 10, 442-8 (2008).

23. Sokumbi O, Wetter DA, Makol A et al. Vasculitis associated with tumor necrosis factor- $\alpha$ inhibitors. Mayo. Clin. Proc. 87, 739-45 (2012).

24. Simard JF, Neovius M, Askling J et al. Mortality rates in patients with rheumatoid arthritis treated with tumor necrosis factor inhibitors: drug-specific comparisons in the Swedish Biologics Register. Arthritis. Rheum. 64, 3502-10 (2012).

25. Piga M, Chessa E, Ibba V et al. Biologics-induced autoimmune renal disorders in chronic inflammatory rheumatic diseases: systematic literature review and analysis of a monocentric cohort. Autoimmun. Rev. 13, 873-9 (2014).

26. Almoallim H, Ghamdi Y, Almaghrabi H. AntiTumor Necrosis Factor- $\alpha$ Induced Systemic Lupus Erythematosus. Open. Rheumatol. J. 6, 315-319 (2012). 